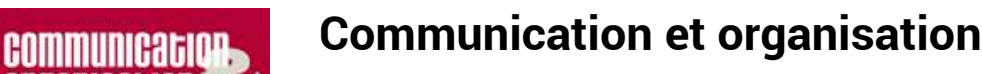

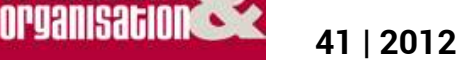

La mutation du métier de communicant public

\title{
Le communicant d'université : un modèle professionnel (dé)bridé
}

Lucia Granget

\section{(2) OpenEdition}

1 Journals

Édition électronique

URL : http://journals.openedition.org/communicationorganisation/3802

DOI : 10.4000/communicationorganisation.3802

ISSN : $1775-3546$

Éditeur

Presses universitaires de Bordeaux

Édition imprimée

Date de publication : 1 juin 2012

Pagination : 181-194

ISBN : 978-2-86781-821-9

ISSN : 1168-5549

Référence électronique

Lucia Granget, «Le communicant d'université : un modèle professionnel (dé)bridé », Communication et organisation [En ligne], 41 | 2012, mis en ligne le 01 juin 2015, consulté le 19 avril 2019. URL : http://

journals.openedition.org/communicationorganisation/3802 ; DOI : 10.4000/

communicationorganisation.3802 


\title{
Le communicant d'université : un modèle professionnel (dé)bridé
}

\author{
Lucia Granget ${ }^{1}$
}

\section{Introduction :}

Pour évoquer la figure de communicant universitaire européen Paolo Pomati, Président de l'Euprio (European Universities Public Relations E Information Officers) fait référence (2009) au film de Pedro Almodovar, "Femmes au bord de la crise de nerfs »! Dans un monde universitaire oscillant entre progrès et crise, entre mission de service public et logique entrepreneuriale, nous proposons d'analyser et de comprendre le chemin parcouru depuis vingt ans par le communicant d'université. S'il fait l'objet d'une classification professionnelle et s'il appartient à des associations d'encadrants de la communication spécifiques à l'enseignement supérieur et à la recherche (ARCESS, COMOSUP, EUPRIO), pourquoi subsiste-t-il encore un flou sur ses fonctions et sur son activité ? En quoi, la donne conversationnelle du web social perturbe mais aussi renforce une forme professionnelle soumise à la « formule de compromis » (Boltanski, 1990) ? Quelle est l'évolution de ce qu'on nommera le « modèle professionnel » (Walter, 1995) des encadrants de la communication d'université, dans le sens d'un agencement arbitraire de données collectées portant, d'une part, sur l'évolution des médias et le contexte organisationnel de l'université, et d'autre part, sur « la compétence possédée et mise en œuvre par le sujet agissant » (Zarifian, 1999) ?

\section{Spécificités de l'étude et aperçus historiques}

\section{Eléments de méthodes}

La recherche entreprise se situe dans le cadre des épistémologies constructivistes (Le Moigne, 1999) avec l'adoption d'une vue « systémique complexe »(Morin, 2005). L’usage de méthodes qualitatives sur une longue

1. Lucia Granget est maître de conférences à l'Université du Sud Toulon-Var et chercheur au laboratoire I3M. Elle est responsable du master «e-rédactionnel». Son activité de recherche est centrée sur la communication des organisations ; granget@univ-tln.fr 
période a été associé à l'approche ouverte de la grounded theory (théorie fondée). Formulée en 1967 par Barney Glaser et Anselm Leonard Strauss (2010), cette approche a permis de faire ressortir des thèmes communs et parfois des contradictions. Elle a également autorisé l'établissement de liens entre la littérature consacrée à l'université, l'analyse des discours officiels et médiatiques portant sur l'enseignement supérieur et la recherche, l'étude des supports de communication émanant des établissements et les données collectées dans le cadre d'entretiens compréhensifs. La grounded theory s'inscrit dans une démarche d'induction analytique. Elle s'élabore selon Jean-Claude Kaufmann (2001) à partir des faits et renverse le rapport au terrain en reliant compréhension et explication. Elle n'impose pas « aux données des présupposés et catégories a priori issus d'un cadre hypothético-déductif, la recherche doit générer une théorie qui "s'ajuste » et " marche » parce qu'elle dérive des concepts et catégories utilisés par les acteurs sociaux eux-mêmes pour interpréter et organiser leur monde » (Jones, 1987).

Des entretiens compréhensifs ont été menés auprès des encadrants des services communication des universités françaises en 2002 et 2004 (20 interviewés), en 2008 (15 interviewés) et 2010 (12 interviewés). L'objectif des trois premières séries d'entretiens $(2002,2004,2008)$ était de mieux comprendre l'évolution de la communication des universités dans un contexte de crise et de changement. Il s'agissait également de mettre en évidence un rapprochement entre communication publique et communication privée. La démarche orientée uniquement vers la diffusion de l'information nécessaire à l'activité de formation et de recherche se révélait inadaptée dans une logique d'autonomie des universités et de système concurrentiel. Les responsables de communication étaient porteurs des valeurs des institutions universitaires et subissaient les effets des changements produits dans un contexte de marchandisation de l'enseignement et de la recherche. Des paradoxes essentiels, ont été établis, comme notamment, "l'incapacité des universités à se positionner dans un environnement concurrentiel en raison de messages analogues, inscrits dans une perspective de contribution au progrès et largement contrecarrés par la médiatisation de tous les maux universitaires et par la mauvaise position des universités françaises dans les palmarès internationaux » (Granget, 2006).

Dans la dernière série d'entretiens compréhensifs (2010), il s'agissait d'interroger les pratiques professionnelles des responsables de communication et d'appréhender l'évolution de ces pratiques face à l'impact des TIC et en particulier du web 2.0. L'énonciation par les communicants de leur vision de la communication des universités a été privilégiée. Les résultats de ces entretiens sont probablement partiels. L'analyse peut avoir privilégié ou négligé certains aspects. Par ailleurs, des données quantitatives ont été recueillies de façon informelle dans le cadre des entretiens menés. Les résultats d'études quantitatives commanditées par l'ARCES $(2009,2011)$ 
sont également exploités car ils permettent de mettre en évidence notamment la féminisation de la profession, des faibles salaires pour un niveau élevé de formation principalement universitaire. Toutefois, la mobilisation de ces résultats statistiques est neutre par rapport l'épistémologie choisie.

La notion de modèle professionnel, ici abordée, «metl'accent sur le processus de construction d'une forme professionnelle...par des professionnels (et non par les seuls experts en sciences sociales). En effet, un modèle est le produit d'expériences, d'observations, de réflexions sur le réel "(Walter, 1995). L'objectif de la démarche engagée est de mettre en évidence les ajustements en cours susceptibles d'expliquer au moins partiellement le fonctionnement de la communication des universités et l'utilisation par les acteurs de dispositifs conceptuels et techniques. Il s'agit également de mettre en mot des réflexions et des opinions qui permettent de révéler des tentatives de mise en œuvre de stratégies identitaires basée sur la définition d'un modèle de compétences. La littérature sur la question de la compétence est abondante. Philippe Zarifian (2004), définit un modèle de la compétence dans lequel « la compétence est la prise d'initiative et l'assumer de responsabilité de l'individu sur des problèmes et évènements auxquels il s'affronte au sein des situations professionnelles $\{\ldots\}$. Pour l'auteur, "l'assumer de responsabilité » signifie " assumer la plénitude de son action » et «manifester le souci d'autrui ». La compétence est, en conséquence, " une logique pratique des situations qui s'appuie sur des connaissances acquises et les transforme avec d'autant plus de force que la diversité des situations augmente $\{\ldots\}$. La compétence est la faculté à mobiliser des réseaux d'acteurs autour des mêmes situations, à partager des enjeux, à assumer des domaines de responsabilités » (Zarifian, 2004).

Enfin, au travers de la nature du travail produit par les équipes en charge de la communication, les universités, dans le cadre de leur mission de service public, se trouvent questionnées sur leurs pratiques d'information, de communication et de médiatisation.

\section{Evolution de la communication et introduction des TIC dans les universités}

Dès le XIII e siècle, l'Université fait l'objet de critiques, crises et conflits. «Depuis l'origine, l'université est toujours apparue en attente d'une nouvelle réforme " (Charle et Verger, 1994). L'Université en France, disparaîtra avec la loi du 15 septembre 1793 et il faudra attendre un décret napoléonien de mars 1808, commémoré comme l'acte fondateur de l'Université, pour définir son architecture et son organisation. L'université est envisagée dans sa fonction utilitariste alors qu'en parallèle, se développent les grandes écoles. Après avoir relevé les enjeux de la massification et de la démocratisation selon le principe de l'égalité pour tous, l'université affronte désormais, ceux de la mondialisation et de l'excellence pour relancer la croissance de la nation.

Depuis les années 80, sous l'effet du mythe de l'entreprise, les services publics ont été amenés à se moderniser. Pour Christine Musselin (2001), c'est 
l'introduction des contrats d'établissement (1989) qui a constitué le tournant significatif permettant une déstabilisation du modèle centralisé uniforme et égalitaire. Le développement d'une politique propre va favoriser la mise en avant des spécificités de chaque établissement. La fonction communication est créée dans les années 1990. "Lidée de développer une grande université bénéficiant d'une image globale devient dépassée au profit de l'émergence d'universités plurielles aux identités institutionnelles plus affirmées» (Musselin, 2001). L'instauration de la réforme du LMD (2002) et l'application de la loi «Libertés et responsabilités » (LRU du 10 août 2007) ont renforcé l'impératif communicationnel. Au fil des rapports officiels relayés par les médias, on peut lire que la France enregistre des résultats médiocres avec pour conséquences, une recherche en déclin, une fuite des étudiants et des enseignants vers les secteurs protégés ou à l'étranger, une sélection par l'échec des étudiants, une dévaluation des diplômes qui conduit à l'allongement des études et au chômage... En réponse, toutes les universités s'emploient à exploiter tous les ingrédients du modèle marketing de la communication : changements de logotypes et de noms, plaquettes, dépliants, insertions publicitaires, création d'événements, diffusion d'objets publicitaires...

Dans les années 90, on assiste à la conception d'une toute première génération de site web dont l'initiative revient principalement aux services informatiques. Les sites sont sommaires. Ils présentent des contenus succincts. Avec l'introduction des TIC dans le supérieur, une seconde génération de site (1998-2000) va naître. Les TIC s'inscrivent « dans un contexte institutionnel en mutation qui vise à faire primer une logique de projet au détriment d'une logique dite de "guichet" "(Barats, Cardy, Thibault, 2002). La fonction communication est présente dans les groupes de pilotage proposés par les services TIC mais elle est rarement porteuse du projet.

Pourtant, se posent les questions essentielles du contenu informationnel et de l'ergonomie. Un travail important de structuration s'impose. La fonction communication s'implique alors fortement. Elle sera amenée à redéfinir les rôles respectifs des outils papier et électronique, de leurs fonctions, de leurs complémentarités et de la nature de leurs contenus. Progressivement, l'intranet de l'internet sera dissocié pour des raisons de confidentialité touchant certaines informations. Les services TIC se structurent et procèdent au recrutement de webmaster. Une troisième génération de site portail va émerger (2005). La recherche par profil pour une information ciblée est alors, d'actualité. Les logiques centrées sur l'utilisateur s'affirment. Des refontes totales ou partielles de sites seront opérées environ tous les trois ou quatre ans. Elles supposent des contenus riches, une diversité d'expression multimédia, une information vivante, l'accessibilité et la navigabilité. La naissance du concept web 2.0 va provoquer, lui aussi, une évolution des pratiques et des champs théoriques de référence dans un univers professionnel déjà mal défini. « Le concept 2.0 évoque un changement de paradigme d'ordre anthropologique dans lequel 
l'intelligence compétitive trouve à la fois de nouveaux outils et un champ d'intervention élargi dans la mesure où la compétitivité est recherchée dans l'ensemble des secteurs d'activité sociale »(Quoniam, 2010).

\section{Le flou de la fonction communication : la technique avant tout, la stratégie malgré tout}

\section{Une fonction hybride :}

L'analyse du phénomène « dircom " par Liliane Messika (1994) et Jacques Walter (1995) montre que "les communicateurs pratiquent un métier dont les contours sont flous ». Selon Catherine Paradeise (1985), tout nouveau groupe professionnel doit soigner son travail argumentaire pour imposer son statut. De plus, les groupes professionnels sont « en lutte continuelle pour maintenir leur place » (Trépos, 1992).

L'encadrant de la communication d'université souffre, en premier lieu, de l'absence de visibilité de son titre. Il est "responsable de la communication", " directeur ", " chargé de communication ", " chargé des relations extérieures" ou de "la communication externe et interne ", parfois "directeur de cabinet " ... La confusion terminologique existe, également, pour désigner l'entité organisationnelle d'appartenance : " service ", " cellule ", " mission ", " division ", "département »... Le terme de " service communication " est toutefois prédominant. Le service est composé de deux à quatre personnes, qui occupent plusieurs fonctions. Le recours à des contractuels et à des prestataires extérieurs renforce le sentiment de "fragilité ». La situation hiérarchique de la fonction communication dans les organigrammes est variable selon les établissements. Elle est révélatrice de l'intérêt que l'on porte à la communication. Le responsable de la communication peut dépendre d'un enseignant-chercheur (Président, vice-président, chargé de mission) ou d'un admininistratif (secrétaire général, directeur des services, chef de cabinet, directeur des ressources humaines pour l'interne). Rattaché à la présidence de l'université, ses missions seront plus politiques. Sous la direction du pôle adminitratif, les tâches seront davantages orientées vers l'information administrative et la communication interne. Les responsables de communication sont principalement des femmes de formation universitaire (majoritairement de niveau bac +5 ). Les responsabilités sont importantes et diversifiées, pour un salaire médiocre, un temps de travail notable, des ressources en personnel limitées et des budgets faibles (ARCES, 2009, 2011). Les associations représentatives des communicants de l'enseignement supérieur et de la recherche cherchent à promouvoir une identité collective mais entretiennent, sans doute, involontairement, une certaine confusion. COMOSUP met en avant (sur son site web), le terme de « responsable de communication des universités ». L'ARCES affiche, celui d'un « responsable de communication de l'enseignement supérieur », à la fois, « directeur de la 
marque ", " dir'com interne ", " directeur de développement » et " manager d'équipe ». EUPRIO propose, celui de "knowledge workers ", pour définir le groupe professionnel en référence aux nouvelles élites organisationnelles anglo-saxonnes. "Le langage comme statut épistémologique » du travail de communication est également posé. Ainsi, la diversité des expressions employées par les associations professessionnelles tendent à maintenir le flou dans la délimitation d'un champ d'intervention composite et difficile à formaliser. Les communicants dénoncent, en effet, une tendance à considérer la communication comme une fonction "fourre-tout » en raison d'objectifs définis par de multiples interlocuteurs et plutôt orientée vers une "prestation de service soumise aux volontés et aux délais des autres ". Les missions sont hybrides car elles couvrent différentes disciplines des Sciences Humaines et Sociales. Les activités multiples relèvent, à la fois, de l'intérêt général dans la dimension diffusion de l'information et des principes de la communication d'entreprise. Le statut de responsable de communication dans un établissement de l'enseignement supérieur et de la recherche se caractérise par une désignation floue de sa profession, de sa qualification et de sa position hiérarchique.

\section{Un rôle polyvalent, stratégique mais surtout technique}

Toute la panoplie des techniques de la communication marketing est exploitée, dans une logique de production de supports et d'actions, un moyen, semble-t-il pour légitimer une fonction qui serait « centrale » mais « dépréciée ». Les plans médias sont privilégiés aux stratégies, la communication externe à la communication interne, l'esthétisme à l'ergonomie et les contenants aux contenus. Les communicants expliquent cette tendance par un système décisionnel interne aléatoire et ambigü. Les décisions importantes sont élaborées, pensées et concertées en dehors des réunions prévues à cet effet au travers d'un réseau informel important et difficile à cerner. L'aspect métaphorique du "modèle décisionnel de la poubelle » (March, 2005) est en vigueur : la décision est le résultat d'une rencontre fortuite entre solutions et problèmes. La communication est alors dans l'incapacité d'anticiper les changements rapides et de fonctionner selon une séquence dictée par une rationalité.Il en est de même au niveau opérationnel.Les unités, les laboratoires, les services, fonctionnent de façon spécifique voire autonome et se mobilisent pour régler les problèmes dans l'urgence... Une forme de résistance culturelle de la communauté universitaire pour valoriser la communication subsiste même si le passage au LMD et l'adoption de la LRU ont provoqué une prise de conscience dans "la nécessité de communiquer ». Cette ambivalence s'explique, d'une part, par l'organisation duelle de l'université qui oppose administration et enseignement/recherche, et d'autre part, par la connotation marketing de la fonction. Issus de formations disciplinaires variées dans le domaine des Sciences Humaines et Sociales, les communicants ne partagent pas forcément une vision commune de la communication publique qui, pour Pierre Zemor 
(2009), ne se réduit pas à la communication concurrentielle : "Dès qu'un responsable de communication parle, il dépend de sa hiérarchie et dépend aussi de son devoir à l'égard des citoyens, il a cette double responsabilité qui forge une véritable déontologie professionnelle, qui peut aboutir à refuser une certaine communication dans l'institution. » Mais, la référence au management, la dimension entrepreneuriale promue par la LRU et les classements peuvent s'avérer contradictoires avec les notions d'intérêt général et de service public d'enseignement et de recherche. Lidée que l'université, voisine comme celle du bout du monde, est une concurrente est désormais très présente dans les esprits. Il s'agit souvent d'attirer le plus d'étudiants possible avec une démarche de marketing de l'offre alors que les étudiants mettent en concurrence les sources d'information et se désintérressent de l'information institutionnelle. Tenus de « respecter certaines règles du jeu » et nécessairement influencés par les réussites du marketing en entreprises, les responsables de communication se construisent un univers symbolique de réussite. L'idée que l'élaboration d'une véritable stratégie de communication établie en concertation avec la direction aura des effets magiques est fortement répandue. En cohérence avec les logiques du branding, les communicants cherchent désormais à valoriser des spécificités et à construire la e-réputation de leur établissement par l'adoption de techniques innovantes.

Lidentité professionnelle des acteurs est déstabilisée dans la représentation professionnelle de leur parcours. A la croisée de plusieurs identités et de multiples pratiques (relations publiques, journalisme d'entreprise, communication, publicité, marketing, intelligence économique...), le communicant explique son action comme celle d'un bâtisseur et d'un défenseur de l'image de son établissement. La communication est présentée comme un cercle vertueux, capable de créer une image de marque, d'attirer les meilleurs étudiants, de lutter contre la concurrence, de convaincre et de générer lien social et motivation,... Face à l'amoncellement de tâches diverses qui envahissent son quotidien, le communicant tente de prendre de la « hauteur». Son travail sur l'image obéirait à une stratégie, à une méthodologie définie et à des actions mesurables. Ainsi, constituée d'un ensemble de techniques directement opérationnelles, la communication marketing aurait réponse à tous les problèmes. Elle constitue, en réalité, une argumentation susceptible de les régler. Dans les faits, les opérations et les messages sont reconduits ou modifiés avec une visée imitatrice, sans véritable analyse et contrôle sur les résultats. Le rôle de relais du discours de la direction semble, même parfois difficile à jouer car la hiérarchie aurait tendance à omettre de transmettre l'information. La fonction de porte-parole de l'institution est limitée. Le communicant pourrait faire l'objet d'une remise en question de la part, notamment, des membres du corps enseignant qui disposent d'une grande liberté de parole. Tout le monde a l'opportunité d'exprimer son point de vue et les journalistes ont l'occasion de se saisir des multiples contradictions pour 
alimenter une polémique existante ou en créer une. De leur côté, les membres de la direction reprocheraient aux responsables de la communication une absence de conseil et de réactivité, principalement, face aux situations de crise. Ainsi, «la communication serait responsable de tous les maux universitaires» alors que les services dédiés s'emploieraient avec obéissance, "à répondre aux sollicitations diverses et variées des acteurs ». Le rôle de la communication interne est également questionné. Faut-il transmettre le discours de la direction ou considérer que la communication interne est le moyen d'expression de l'ensemble des personnels? Dans les faits, c'est le discours officiel qui s'impose et la communication interne est souvent délaissée.

\section{Web 2.0 et prégnance de "l'infobésité "}

\section{Vers l'émergence d'une fonction de médiation}

Le phénomène 2.0 " traduit une société où les échanges sont plus horizontaux, en réseau, et dans laquelle la hiérarchie verticale n'existe plus » (Quoniam, 2010). Les responsables de communication adaptent leur modèle de compétence aux évolutions techniques. La position dans les moteurs de recherche et le web 2.0 sont devenus d'ardentes obligations. Ils travaillent en relation avec un webmaster ou avec un chef de projet multimédia et assurent la gestion éditoriale du site web et du blog avec la volonté de proposer une information complète et claire. Ils ont réussi à suplanter les informaticiens en charge dans les années 90 de la conception de la première génération de sites web. Ils évoquent, aujourd'hui encore, toutefois l'obsolescence de leur site : "décalé par rapport aux objectifs de communication", "peu en phase avec la cible ", "retard technique ", "graphisme et ergonomie dépassés", "interactivité faible ", "absence de scénario de navigation", "version ou application mobile inexistante »... L'intranet reste un levier exploité principalement par les services administratifs et les ressources humaines. La communication interne se résume à la réalisation de newsletters, à l'organisation d'évènements festifs alors que les attentes du personnel sont «nombreuses» et que les demandes des directions d'établissements sont «imprécises » ou « mal formulées».

Avec le web 2.0, la tâche est complexe. Les étudiants mais également le personnel, sont en effet en capacité de publier des informations erronées, d'émettre des avis et de créer un buzz négatif. La crainte de l'opinion hostile et de la crise médiatique qui en découle, est source de tensions. Les responsables de communication se sentent assez démunis face à l'information négative et à la rumeur. Le rôle d'infostratège se développe. Il nécessite d'allier des compétences techniques, juridiques et communicationnelles pour procéder à un patient travail de nettoyage des traces numériques éditoriales négatives et pour actionner les rouages de la construction de la e-réputation. L'obtention d'une visibilité maximale est une priorité avec pour contrepartie le risque de créer une image ou une identité numérique fragmentée. Le communicant 
devient alors community manager car "il est indispensable pour les universités d'être présentes sur les réseaux sociaux » et d'animer sur Facebook une page promotionnelle, d'ouvrir un compte Twitter, de publier des vidéos sur Youtube et Dailymotion (extraits de cours, d'évènements...), de maintenir des relations avec les entreprises avec Linkedln, Viadéo et d'investir Wikipédia en s'appuyant sur les enseignants. Les notions de dialogue, de partage et de relation propres aux réseaux sociaux ne vont pas sans difficulté et la « débrouille » est de mise car il s'agit de maitriser une communication «effrénée» et «spontanée». Plusieurs entités en interne (composantes, services, associations,...) peuvent être amenées à ouvrir des comptes en réponse aux usages sur les réseaux sociaux (regroupement par centres d'intérêts). Toutefois, l'absence de maîtrise de cette inflation d'ouverture de comptes peut produire des dissonances identitaires et multiplier les sources d'expression de mécontentement. Inquiets, les responsables de communication, gardiens de l'image de l'institution, aspirent à la maîtrise de la surcharge informationnelle. La démarche de segmentation est mise en pratique, pour tenter d'identifier des communautés existantes. Le marketing communautaire est sollicité pour tenter de mettre en place des communautés d'anciens étudiants, le marketing de l'attention pour tenter d'humaniser la relation, le marketing conversationnel pour tisser des relations. Le storytelling a été introduit pour raconter les histoires universitaires et professionnelles des anciens. Les contraintes éditoriales sont démultipliées. Le feed-back (veille, référencement, statistiques) fait désormais partie des pratiques mais les analyses sémantiques et sémiotiques sont inexistantes. L'exploitation du web, par les journalistes en quête d'informations inédites et de témoignages authentiques, déstabilise le modèle classique des relations presse. La mise en place d'un marketing d'influence s'impose avec la nécessité de consacrer du temps et de l'énergie pour activer des leaders d'opinion (relais) sur différentes plates-formes (blogs, forums, sites spécialisés), proposer du contenu unique en faisant appel aux communautés et échanger du contenu contre lisibilité et trafic. Mais globalement les méthodes relèvent du "tâtonnement ", de "l'expérience ", de la "patience ", de la "volonté » et de "l'implication de chacun». Ainsi, l'exploitation des potentialités du web 2.0 peut se limiter à " copier/coller » des pages de sites internet sur les réseaux sociaux et à mesurer le nombre de fans sur une fan-page ou d'abonnés sur un compte. Face au web 2.0, les attitudes sont diverses. Elles peuvent être positives et susciter l'adhésion, la curiosité ou encore l'humour. Les dimensions de dialogues, d'échanges, de mise en relation, de création de lien sont promues. Les relations humaines seraient enfin devenues une priorité. L'usager serait au cœur des dispositifs numériques. La communication de masse et les théories de l'information linéaires ne seraient plus d'actualité. Mais les réactions peuvent aussi être négatives : distance, mépris et rejet. La multiplication des outils provoque un effet de "collectionnite ". "La présence de l'Université sur Facebook n'est pas en 
phase avec son identité institutionnelle ». L'utilisation permanente d'anglicismes rend le web 2.0, "indigeste ». La communication a pour rôle "d'insuffler une dynamique pour que les dispositifs collaboratifs initiés par l'université soient davantage utilisés». Mais la mobilisation des acteurs en interne est difficile. L'université n'est pas un collectif homogène et les outils collaboratifs ne sont pas forcément en adéquation avec les besoins. La diffusion de l'information en direction des étudiants et des lycéens est également préoccupante. Ils vivent dans l'ère du nomadisme, détournent les outils pour satisfaire leurs besoins. Ainsi, les logiques institutionnelles sont percutées. Linstitution perdrait en légitimité et en autorité. Toutefois, les avis convergent sur un point : le véritable changement se situe dans les aspects conversationnels du web social. L'appréciation des étudiants (échanges, évaluations positives, dénigrements, manifestations d'humeurs ...) sur les réseaux sociaux, engendre des obstacles d'ordre psychologique, culturel, idéologique et organisationnel. L'avis de l'internaute est perturbant car il est devenu un facteur important de jugement (vie universitaire, formation, contenu des enseignements ...). Le mode de raisonnement des responsables de communication est ébranlé et les solutions envisagées reposent sur la subjectivité. La perte de temps dans l'analyse est source de stress et de surcharge cognitive. L'impérieuse nécessité de la visibilité et de l'influence sur le web conduit à la surenchère permanente des actions et des dispositifs avec la conscience que le contenu juste, véridique et vérifié n'est pas forcément le plus visible. Pourtant, le communicant est appelé à faire des choix, s'il veut être capable de surmonter l'infobésité. Le web 2.0 assigne ainsi, un objectif supplémentaire, fondé sur la prise en considération de l'opinion (interne et externe) et une contrainte additionnelle, "l'impossibilité de travailler déconnecté ». La fonction communication aurait, de plus en plus, une visée consensuelle basée sur le collaboratif qui intègre une dimension de médiation. Jean Davallon (2003), avance l'hypothèse que «le recours à la médiation est effectué lorsqu'il y a mise en défaut ou inadaptation des conceptions habituelles de la communication : la communication comme transfert d'information et la communication comme interactions entre deux sujets sociaux. \{...\} La médiation rend possible l'échange social, alors même que les univers de la production et de la réception sont a priori par nature disjoints $\{\ldots\} »$.

\section{Un communicant bridé pour un modèle débridé de compétences :}

La définition de l'action du communicant a un cadre flou, mouvant et fortement influencé par les tendances du moment. Le mimétisme est le mécanisme dominant. Le communicant est bridé dans son action de différenciation alors que dans le champ du marketing prédomine les notions de positionnement et d'image de marque. Sa marge de manœuvre effective est limitée car il se trouve confronté à un réseau complexe d'acteurs (président, secrétaire général, vice-présidents, chargés de missions, directeurs d'unités, de 
laboratoires et de formations, membres des conseils, chefs de services ...) qui vont influencer et contribuer à la prise de décision. Alors la tentation est grande de s'inspirer de ce qui s'est déjà fait ou de ce qui se fait ailleurs. Les missions confiées sont vagues malgré l'existence de fiche de poste qui reflète rarement l'étendue de l'activité de communication. La démonstration par l'exemple, est, l'un des meilleurs moyens pour convaincre dans un contexte de plus en plus marqué par les logiques de benchmarking et de ranking. Les actions de communication mises en œuvre subissent l'influence de l'hypermédiatisation des palmarès des établissements d'enseignement supérieur. Les pratiques évaluatrices qui s'appuient sur la nécessité d'informer les citoyens sur les choix opérés en matière d'enseignement et de recherche participent à la volonté de créer une image de performance. L'évaluation devient " porteuse d'un projet stratégique » et l'occasion de partager une prétendue " histoire collective ». La communication est inspirée par " la vision du monde » portée par l'évaluation. Le langage recommandé par le New Management Public, est adopté. Peu d'universités françaises sont parvenues à devenir des marques. Leur visibilité et leur réputation semblent être, désormais, corrélées à l'idée de reconnaissance par l'évaluation et les classements. Pour certains des communicants interrogés, l'université aurait perdu, paradoxalement, de son prestige en tentant de répondre aux exigences de la société. L'approche stratégique dépend, au final, de la capacité du responsable de communication à argumenter des choix issus de ses propres perceptions, de la conjoncture et de son propre réseau relationnel. L'activité se définit en fonction de la personnalité, de la formation, du parcours professionnel, de la relation avec la hiérarchie, de la capacité à prendre des décisions et par rapport aux usages véhiculés par le groupe professionnel. Le champ professionnel souffre d'imprécisions et l'identité professionnelle des communicants s'affirme avec difficulté. Chaque acteur affirme son appartenance à un métier dont la désignation est variable selon l'établissement. Les responsables de communication sont à la recherche de légitimité. Ils ressentent un certain scepticisme autour de leur action de la part des personnels de l'université. Leur utilité sociale a toujours fait l'objet de controverse en raison d'une activité marquée par le marketing hérité de la sphère marchande, par une filiation avec les relations publiques porteuses d'une connotation de superficialité et par le modèle guerrier de la stratégie revendiqué pourtant avec force. La transformation des dispositifs de communication et le concept 2.0 ont entraîné le communicant universitaire, dans la voie de la médiation, même s'il éprouve encore des difficultés à se dessaisir d'une vision centralisatrice. L'agir professionnel croise désormais plusieurs domaines interdisciplinaires qui peuvent être interprétés comme modèles communicationnels et informationnels en référence à l'information de nature publique (journalistique), en liaison avec le marketing (publicitaire, évènementiel, d'influence, relationnel, alternatif, branding et fundraising comme conséquence de la LRU), en relation avec la communication des 
organisations (SIC, sociologie, anthropologie, psychologie sociale...), en lien avec les technologies de l'information et de la communication (formalisation de la ligne éditoriale, écriture multimédia, indexation et référencement éditorial mais aussi participation à l'ergonomie, à l'architecture de l'information, au design, à l'accessibilité...) et en rapport avec l'intelligence compétitive (activités de veille et d'information). Le web 2.0 inscrit le modèle professionnel dans une phase relationnelle avec pour limite l'illusion de la modernité et la nécessité d'établir une relation de confiance avec le public. Une communication directe et frontale cohabite désormais avec la mission de transmission de la voix officielle. Si les acteurs interagissent entre eux, un jeu de rôle semble s'instaurer. Il allie la confiance dans la relation de proximité et la défiance envers le collectif. Le responsable de communication a désormais intégré les fonctions d'infostratège et de community manager. Il envisage son rôle comme celui, d'une part, d'un veilleur et d'autre part, d'un animateur, d'un tuteur et d'un relationniste, ayant à la fois, la charge de rechercher et traiter l'information mais aussi celle, de stimuler les échanges, d'accompagner l'usager, pour parvenir à influencer l'opinion. La fonction éditoriale tend, de fait, à se renforcer, elle est un cadre pour éviter les débordements liés à la profusion des dispositifs transversaux. Les responsables de communication sont perplexes ou désorientés. Ils revendiquent leur appartenance à métier qui nécessiterait selon eux, d'être enfin reconnu au sein de leur établissement et par les personnels. La participation à des réunions et notamment à celles du comité des directeurs procure une certaine lisibilité à la fonction. Les communicants expriment, également, le besoin de créer et d'innover. Ils sont en attente d'une reconnaissance au moins pour la partie de leur travail mesurable en quantité de production. Le temps consacré à l'analyse et à la réflexion est largement méconnu. Le dévouement à l'institution est ignoré. Face à la montée en puissance des tâches et des attentes, ils ont l'impression que l'absence de considération s'accentue. Cette perception, peut parfois entraîner des frustrations ou des ressentiments qui peuvent conduire au désinvestissement. Mais au-delà, les responsables de communication ont l'impression d'appartenir à un groupe hybride et presque à l'écart du monde universitaire.

\section{Conclusion}

La communication des universités est composite et l'identité professionnelle des acteurs est instable, métissée et évolutive. Le web 2.0 et la prégnance de "l'infobésité » accentuent la forme débridée du modèle de compétences des responsables de communication d'université. Ce modèle est interdisciplinaire et fait cohabiter de multiples registres d'actions. La juxtaposition d'un amalgame de tâches et d'activités qui relèvent de la communication et de l'information, permet de mettre en évidence un fonctionnement acrobatique. Dans un contexte d'évolutions techniques et organisationnelles, l'expertise du modèle 
professionnel d'encadrement de la communication se trouvent en tension face à la nébuleuse croissante de méthodes et de savoirs opérationnels à maîtriser. La conception de la communication continue à être unidirectionnelle malgré la perte du monopole discursif. Avec le web social, le schéma communicatif n'est plus totalement maîtrisé et les risques de crise d'image tendent à se développer. L'environnement numérique oblige le communicant à adopter une attitude de professionnel-citoyen qui conçoit son action, sans artifice et faux semblant, dans une proximité réelle avec un internaute libre de ses choix.

\section{BIBLIOGRAPHIE}

BARATS C., CARDY H., THIBAULT F., " Le discours "institutionnel" d'introduction des TIC dans l'enseignement supérieur français : écrire/ s'inscrire dans l'innovation », Marseille, actes du XIII ${ }^{e}$ Congrès national de la SFSIC, 2002.

BOLTANSKI L., La sociologie des professions, Encyclopédie philosophique, Paris, PUF, 1990.

CHAUDY, S., GRANGET, L., Réputation des territoires et des organisations 2.0, dans QUONIAM L., LUCIEN A. (sous la direction de), Intelligence compétitive 2.0, Paris, Hermes Lavoisier, 2010.

DAVALLON J., " La médiation : la communication en procès ? ", MEI, 2004, nº 19 .

GLASER B., STRAUSS A.L., La découverte de la théorie ancrée, Paris, Essai, 2010.

GRANGET L. « Modèles et réalités incertaines de l'université ", Communication \& Organisation, n³0, 2006,.

JONES S., " Choosing Action Research : a Rationale » in MANGHAM I.L. (ed.), Organization Analysis and Development, Chichester, Wiley, 1987.

MESSIKA L., " Dircoms et journalistes : une convergence du flou ", Réseaux, 1994, $n^{\circ} 64$.

LE MOIGNE J-L., Les Épistémologies Constructivistes, Paris, Que sais-je ?, 1999.

MARCH, J.G., «Technologie de la déraison et éloge de la lenteur », propos recueillis par Jardin E., Les Organisations, Paris, Sciences Humaines, 2005.

MORIN E., Introduction à la pensée complexe, Paris, Essai, 04/2005.

MUSSELIN C., La longue marche des universités françaises, Paris, PUF, 2001.

KAUFMANN J-C, L'entretien compréhensif, Paris, Nathan, 2001.

PARADEISE C., "Rhétorique professionnelle et expertise ", Sociologie du travail, $1985, n^{\circ} 1$.

TRÉPOS J-Y., Sociologie de la compétence professionnelle, Nancy, Presse Universitaire de Nancy, 1992.

QUONIAM L. Introduction, dans QUONIAM L., LUCIEN A. (sous la direction de), Intelligence compétitive 2.0, Paris, Hermes Lavoisier, 2010.

STRAUSS A., La trame de la négociation. Sociologie qualitative et interactionnisme, Paris, L'Harmattan, 1997. 
WALTER J., Directeur de communication, Paris, L'Harmattan, 1995.

ZARIFIAN P., Le modèle de la compétence, Paris, Editions Liaisons, 2004.

ZEMOR P., «Entretien avec Pierre Zemor », Communication E̊ Organisation, n³5, 2009.

Résumé : Cet article tente de saisir et de caractériser le modèle de compétence de l'encadrant de la communication au sein des universités. Ce modèle est interdisciplinaire et fait cohabiter de multiples registres d'actions. Dans un contexte de plus en plus marqué par les logiques de benchmarking et de ranking, le communicant est bridé dans la mise en œuvre de son rôle stratégique qu'il considère pourtant au cœur de son activité. Le web 2.0 et la prégnance de "l'infobésité » provoquent, en contraste, une forme débridée de son modèle de compétences. A la croisée de plusieurs pratiques, l’identité professionnelle des acteurs est instable, métissée et évolutive.

Mots-clés : Community manager, identité professionnelle, infobésité, modèle de compétence, responsable de communication, stratégie de communication, web 2.0.

\begin{abstract}
This article attempts to capture and characterize the competency model of framing communication in universities. This model is interdisciplinary, multiple and shares registers of actions. In an environment increasingly marked by the logic of benchmarking and ranking, the communicator is clamped in the implementation of its strategic role, however, he considers the heart of its business. Web 2.0 and the significance of "information overload" causing, in contrast, an unbridled form of a competency model. At the crossroads of several practices, the professional identity of actors is unstable, mixed and scalable.
\end{abstract}

Keywords : Community manager, professional identity, competency model, framing communication, information overload, web 2.0. 